University of Wollongong

Research Online

Australian Institute for Innovative Materials -

Papers

Australian Institute for Innovative Materials

$1-1-2014$

One-step synthesis of graphene/polypyrrole nanofiber composites as cathode material for a biocompatible zinc/polymer battery

Sha Li

University of Wollongong, sl739@uowmail.edu.au

Kewei Shu

University of Wollongong, ks323@uowmail.edu.au

Chen Zhao

University of Wollongong, cz995@uowmail.edu.au

Caiyun Wang

University of Wollongong, caiyun@uow.edu.au

Zaiping Guo

University of Wollongong, zguo@uow.edu.au

See next page for additional authors

Follow this and additional works at: https://ro.uow.edu.au/aiimpapers

Part of the Engineering Commons, and the Physical Sciences and Mathematics Commons

Research Online is the open access institutional repository for the University of Wollongong. For further information contact the UOW Library: research-pubs@uow.edu.au 


\title{
One-step synthesis of graphene/polypyrrole nanofiber composites as cathode material for a biocompatible zinc/polymer battery
}

\author{
Abstract \\ The significance of developing implantable, biocompatible, miniature power sources operated in a low \\ current range has become manifest in recent years to meet the demands of the fast-growing market for \\ biomedical microdevices. In this work, we focus on developing high-performance cathode material for \\ biocompatible zinc/polymer batteries utilizing biofluids as electrolyte. Conductive polymers and graphene \\ are generally considered to be biocompatible and suitable for bioengineering applications. To harness the \\ high electrical conductivity of graphene and the redox capability of polypyrrole (PPy), a polypyrrole fiber/ \\ graphene composite has been synthesized via a simple one-step route. This composite is highly \\ conductive $\left(141 \mathrm{~S} \mathrm{~cm}^{-1}\right)$ and has a large specific surface area $\left(561 \mathrm{~m}^{2} \mathrm{~g}^{-1}\right)$. It performs more effectively \\ as the cathode material than pure polypyrrole fibers. The battery constructed with PPy fiber/reduced \\ graphene oxide cathode and $\mathrm{Zn}$ anode delivered an energy density of $264 \mathrm{mWh} \mathrm{g}^{-1}$ in $0.1 \mathrm{M}$ phosphate- \\ buffer saline.

\section{Keywords} \\ composites, nanofiber, polypyrrole, graphene, synthesis, step, one, zinc, biocompatible, battery, material, \\ polymer, cathode \\ Disciplines \\ Engineering | Physical Sciences and Mathematics

\section{Publication Details} \\ Li, S., Shu, K., Zhao, C., Wang, C., Guo, Z., Wallace, G. \& Liu, H. Kun. (2014). One-step synthesis of \\ graphene/polypyrrole nanofiber composites as cathode material for a biocompatible zinc/polymer \\ battery. ACS Applied Materials and Interfaces, 6 (19), 16679-16686.
}

\section{Authors}

Sha Li, Kewei Shu, Chen Zhao, Caiyun Wang, Zaiping Guo, Gordon G. Wallace, and Hua-Kun Liu 


\title{
One-step synthesis of graphene/polypyrrole
}

\section{nanofibre composites as cathode material for}

\section{biocompatible zinc/polymer battery}

\author{
Sha $\mathrm{Li}^{1,2}$, Kewei Shu ${ }^{2}$, Chen Zhao ${ }^{2}$, Caiyun Wang**2, Zaiping Guo ${ }^{1}$, Gordon Wallace ${ }^{2}$, Hua Kun \\ $\operatorname{Liu}^{* 1,2}$, \\ ${ }^{1}$ Institute for Superconducting and Electronic Materials, University of Wollongong \\ ${ }^{2}$ ARC Centre of Excellence for Electromaterials Science, University of Wollongong, \\ AIIM Facility, Innovation Campus, \\ North Wollongong, NSW 2500, Australia
}




\section{ABSTRACT}

The significance of developing implantable, bio-compatible, miniature power sources operated in a low current range has become manifest in recent years to meet the demands of the fast growing market for biomedical microdevices. In this work, we focus on developing high performance cathode material for biocompatible zinc/polymer batteries utilizing biofluids as electrolyte. Conductive polymers and graphene are generally considered to be biocompatible and suitable for bioengineering applications. To harness the high electrical conductivity of graphene and the redox capability of polypyrrole (PPy), a polypyrrole fibre/graphene composite has been synthesized via a simple one-step route. This composite is highly conductive $\left(141 \mathrm{~S} \mathrm{~cm}^{-1}\right)$ and has a large specific surface area $\left(561 \mathrm{~m}^{2} \mathrm{~g}^{-1}\right)$. It performs more effectively as the cathode material than the pure polypyrrole fibres. The battery constructed with PPy fibre/reduced graphene oxide (RGO) cathode and $\mathrm{Zn}$ anode delivered an energy density of $264 \mathrm{mWh} \mathrm{g}^{-1}$ in $0.1 \mathrm{M}$ phosphate buffer saline.

KEYWORDS: biobattery, polypyrrole fibre/graphene composite, simulated body fluid, aqueous zinc-air battery, polymer cathode

\section{INTRODUCTION}

Implantable medical devices (IMDs), such as cardiac pacemakers ${ }^{1-3}$, defibrillators ${ }^{4}$, implantable monitors and imaging devices ${ }^{5-6}$, cochlear implants ${ }^{7}$, etc., are applied universally in modern clinical diagnosis and treatment. Generally, the IMDs are powered by independent battery systems providing stable and continuous electrical energy output ${ }^{8}$. The current batteries 
for IMDs mainly include various types of lithium batteries, including $\mathrm{Li} / \mathrm{I}_{2}-\mathrm{PVP}$, where PVP is poly 2-vinyl pyridine, $\mathrm{Li} / \mathrm{V}_{2} \mathrm{O}_{5}, \mathrm{Li} / \mathrm{MnO}_{2}, \mathrm{Li} / \mathrm{SVO}$, where SVO is silver vanadium oxide, $\mathrm{Li} / \mathrm{SO}_{2}$ 2, 9-10, and zinc-air batteries for hearing-aid devices ${ }^{7,11}$. These batteries are generally designed to power therapeutic devices for chronic diseases (e.g., cardiac pacemakers) because of their high discharge voltage and high energy density.

More recently, enormous research work has been carried out to develop microscale implantable medical devices (mIMDs), including implantable diagnosis/monitoring capsules ${ }^{12}$, microscale drug pumps ${ }^{13}$, implantable microsensing devices ${ }^{14-15}$, etc. These devices usually require flexible, microsized, and even non-toxic, implantable power sources that can be easily integrated into the microdevices without inducing a significant size/weight increment. Traditional implantable power sources, including the lithium batteries and zinc-air batteries, all require total encapsulation by stiff metal shells to prevent the leakage of toxic substances. This inevitably confines their shapes and produces extra weight. Therefore, it is necessary to explore new types of implantable power sources that can be specified for powering those advanced mIMDs.

Miniature or flexible aqueous metal-air batteries are currently considered to be one of the most promising candidates for powering mIMDs, which mainly include the zinc-air battery system and the magnesium-air battery system ${ }^{11,16-17}$. The volume of these batteries can be easily reduced by reforming the battery into one monolithic flexible structure with a non-toxic and ductile metal anode ${ }^{18-19}$. Meanwhile, body fluids such as blood plasma, gastric fluid, or urine are all ionic 
conductive solutions that can be applied as electrolyte $\mathrm{e}^{20-22}$. Therefore, it is possible for the battery to be dry-implanted into the human body when sealed by water-permeable films.

Compared to the Mg-air battery, the Zn-air battery has predominant advantages, since the interface reaction of the $\mathrm{Zn}$ anode is more controllable in aqueous electrolyte ${ }^{23}$. The aqueous zinc-air battery is powered by the redox reaction between the zinc anode and oxygen, while its cathode material usually works as the oxygen reduction catalyst, which provides oxygen ions for zinc oxidation throughout the discharge process (as illustrated in Figure 1$)^{24-25}$. The electrocatalytic activity of the cathode material is critical to the battery performance ${ }^{26}$. To find the ideal cathode material is a top priorityin designing such a battery.

Conductive polymers (CPs) are considered to be ideal cathode materials for a biocompatible zinc-air battery, since they are non-toxic, electrically conductive, and redox active ${ }^{25,} 27-28$. Recently it was found that graphene is a metal-free catalyst for the oxygen reduction reaction $(\mathrm{ORR})^{29-32}$. A superior performance may be expected from a conducting polymer-graphene composite in the Zn-air battery. In this work, nanostructured polypyrrole is designed to be incorporated into a composite with graphene nanosheets. This composite is obtained via a simple one-step synthesis route and applied as a cathode material coupled with zinc anode in simulated body fluid and $0.2 \mathrm{M}$ phosphate buffered saline. The discharge performance of this type of battery suggests its prospects as a promising power source for mIMDs. 


\section{EXPERIMENTAL}

\subsection{Materials preparation}

Chemicals used for materials synthesis were all purchased from Sigma Aldrich Australia Pty Ltd. The polypyrrole fibre is synthesized via a chemical polymerization method using cetrimonium bromide (CTAB) as a soft template. Pyrrole was freshly distilled prior to use. 0.109 g (0.3 mM) CTAB was added into $200 \mathrm{ml} \mathrm{N}$-saturated $0.1 \mathrm{M}$ hydrochloride acid solution to form spherical micelles for wrapping the pyrrole monomers ${ }^{33}$, followed by the addition of 0.27 $\mathrm{ml}$ (20 mM) pyrrole. The pyrrole/CTAB suspension was stirred and placed in an ice-water bath to keep the reaction temperature at $0{ }^{\circ} \mathrm{C} .0 .91 \mathrm{~g}(20 \mathrm{mM})$ ammonium persulfate was dissolved in $10 \mathrm{ml}$ water and added dropwise into the suspension. The mixture was stirred for 24 hours for complete polymerization. The as-prepared polypyrrole fibre was washed with deionized water and ethanol to remove the surfactant residuals and then vacuum dried at $60^{\circ} \mathrm{C}$ for 12 hours.

The polypyrrole fibre/graphene composite was synthesized via a one-step polymerization method using pyrrole monomers and graphene oxide (GO) aqueous solution as precursors. Graphene oxide was obtained from natural graphite powder by a modified Hummers method through the exfoliation process developed by Xu et al. ${ }^{34}$. The concentration of the as-prepared graphene oxide solution was quantified as $9.6 \mathrm{mg} \mathrm{ml}^{-1} .2 \mathrm{ml} \mathrm{GO}$ solution was diluted by $20 \mathrm{ml}$ (0.1 M) hydrochloric acid solution and then mixed with pyrrole (20 mM) and CTAB (1.5 mM). The same polymerization procedure was followed as for polypyrrole fibres. The resulting powder was filtered and washed with ethanol and deionized water, and then stirred with $100 \mathrm{~mL}$ (2 M) ascorbic acid solution at $60^{\circ} \mathrm{C}$ for 5 hours to reduce the GO component. The reduced 
composite was washed with deionized water and ethanol to remove the residuals and then vacuum dried at $60{ }^{\circ} \mathrm{C}$ for 12 hours.

\subsection{Battery construction}

A smooth slurry containing $80 \%$ (wt.\%) active material, 10\% polyvinylidene fluoride (PVDF) and $10 \%$ carbon black was prepared in a KK-250S planetary mixer (Mazerustar, Japan). The cathode electrodes were fabricated by pasting the composite slurry onto a square piece of stainless steel mesh with an area of $1 \mathrm{~cm}^{2}$. Pieces of zinc foil were polished, ultrasonically cleaned with ethanol, and cut into $1 \mathrm{~cm} \times 2 \mathrm{~cm}$ pieces. The battery was tested in home-made glass cells with a fixed width of $0.5 \mathrm{~cm}$ (Figure 2). Both the cathode and the anode were attached tightly to the cell wall, and simulated body fluid was injected just before testing. The electrolyte, namely, the revised simulated body fluid (r-SBF), which has a similar ionic composition to human blood plasma (Table 1), was prepared by the procedure introduced by Tadashi Kokubo et al. ${ }^{35}$..

\subsection{Characterizations}

The chemical properties of polypyrrole (PPy) fibre and PPy fibre/reduced graphene oxide (RGO) composite were investigated by both Raman spectroscopy (JOBIN YVON HR800 Confocal Raman system) and X-ray photoelectron spectroscopy on a system equipped with a SPECS PHOIBOS 100 Analyser and an $\mathrm{Al} \mathrm{K \alpha}$ radiator as the X-ray excitation source. Their morphologies were investigated by field emission scanning electron microscopy (FESEM; JOEL 
7500) and transmission electron microscopy (TEM; JOEL JEM-2011). The surface areas of materials were determined with a Nova 1000 Brunauer-Emmett-Teller (BET) surface area analyser, and their conductivities were measured with a JANDEL RM3 four-point-probe instrument. Cyclic voltammetry (CV) and electrochemical impedance spectroscopy (EIS) were performed on a CHI 660 electrochemistry workstation. The battery discharge performances were measured with a Land ${ }^{\mathrm{TM}}$ battery testing system.

\section{RESULTS AND DISCUSSION}

3.1 Characterizations of cathode materials

The concentration of CTAB was strictly controlled at 4 times its critical micelle concentration (CMC) in the polypyrrole synthesis. Under this condition, the formed micelles are wire-like. It was expected that pyrrole monomers would be encapsulated in the hydrophobic cores of the surfactant micelles during the polymerization process and that the formed polypyrrole would keep the same morphology as that of the micelles. The polypyrrole obtained in this work is in fibre form, and these fibres are long and uniform, with an average thickness of $100 \mathrm{~nm}$ [Figure 3(a)]. The BET specific surface area of the PPy fibres was determined to be $345 \mathrm{~m}^{2} \mathrm{~g}^{-1}$, and its electric conductivity was measured as $76.4 \mathrm{~S} \mathrm{~cm}^{-1}$.

After being incorporated with the RGO nanosheets, these polypyrrole fibres are interwoven between graphene layers with a relatively smaller average diameter, which is approximately 70 $\mathrm{nm}$ [Figure 3(b)]. Such a structure is much clearer under TEM [Figure 3(d)]. The long and tangled polypyrrole fibres are evenly distributed on the RGO nanosheets, and the RGO sheets are 
coated with a sheer layer of the amorphous polypyrrole. The large-scale two-dimensional coated graphene sheets decorated with fine polypyrrole fibres possess a larger specific surfaces area than that of the polypyrrole fibres. Not surprisingly, both the specific surface area and the conductivity of the composite are significantly increased to $561 \mathrm{~m} \mathrm{~g}^{-1}$ and $141 \mathrm{~S} \mathrm{~cm}^{-1}$, respectively. Both the large surface area and the high electrical conductivity are crucial for a cathode material to achieve high electrochemical catalytic activity.

To characterize the chemical structure of the composites after reduction and the interactions between the polypyrrole fibres and the graphene nanosheets, Raman spectroscopy was performed on each sample [Figure 4(a-c)]. All of the samples display characteristic peaks that are assigned to the doped polypyrrole structures ${ }^{36}$. The peak representing the $\mathrm{C}-\mathrm{H}$ in-plane deformation appears at $934 \mathrm{~cm}^{-1}$ for pure polypyrrole fibres, but it is shifted to $934 \mathrm{~cm}^{-1}$ for PPy/GO and 927 $\mathrm{cm}^{-1}$ for PPy/RGO. Meanwhile, small shoulder peaks adjacent to the $930 \mathrm{~cm}^{-1}$ band are observed in the spectra of both the PPy/RGO $\left(985 \mathrm{~cm}^{-1}\right)$ and the PPy/GO $\left(981 \mathrm{~cm}^{-1}\right)$. These peaks are related to the reduced state of the PPy chain $^{37}$, suggesting that the PPy contained in both of the composites, which is composed of cation doped and dication doped states, is not fully oxidized. The Raman spectra also reveal that the functional groups on the graphene basal plane are associated with the nitrogenous functional groups of the PPy backbone via the same doping mechanism as that of $\mathrm{Cl}^{-}$, although with slightly less doping efficiency ${ }^{38}$. The graphene basal structure has two well documented characteristic peaks, including the D-band at $1340 \mathrm{~cm}^{-1}$ and a broad G-band at $1580 \mathrm{~cm}^{-1}$ [Figure 4(c)]. These two peaks correspond to the first order scattering of the $E_{2 g}$ mode of the graphite lattice and the in-plane bond stretching motion of the carbon $s p^{2}$ atoms, respectively ${ }^{39}$. The $2 \mathrm{D}$ peaks are also notable in both the RGO and GO spectra, indicating 
that the thickness of the exfoliated graphene oxide is acceptable. The D band and the G band regions are broadened for both the PPy/GO and the PPy/RGO composites, as they overlap with the ring stretching $\left(1325 \mathrm{~cm}^{-1}\right)$ and C-C backbone $\left(1600 \mathrm{~cm}^{-1}\right)$ stretching band regions of polypyrrole. This also provides evidence for the strong chemical interactions between graphene and polypyrrole. The intensity ratio of the $G$ band to the $D$ band is significantly higher for PPy/RGO composite $\left(I_{G}: I_{D}=1.1\right)$ compared to that of the PPy/GO composite $\left(I_{G}: I_{D}=0.83\right)$. This is mainly due to the emergence of structural defects after the removal of large amounts of oxygen functional groups ${ }^{40-41}$. It is notable that ascorbic acid also slightly reduces the polypyrrole, causing the splitting of the band in the $1580 \mathrm{~cm}^{-1}$ region. The reduction of polypyrrole, however, has almost no negative effects on the electrical conductivity of the PPy/RGO composite, since the high electrical conductivity of the composite is mainly contributed by the fast electron transfer rate of the reduced graphene oxide.

The reduction effect of ascorbic acid on the PPy/RGO composite was further investigated by X-ray photoelectron spectroscopy (XPS), as shown in Figure 5. The peak deconvolution and fittings were carried out using Gaussian-Lorentzian shaped peaks based on the Shirley background correction. The peak at $284.5 \mathrm{eV}$ for the C 1s spectra represents non-oxygenated C$\mathrm{C}$ bonding, which is intensified after reduction. The broad peak at $288.4 \mathrm{eV}$ can be assigned to the carbon-oxygen bonding, including $\mathrm{C}-\mathrm{O}$ and $\mathrm{C}=\mathrm{O}^{42}$. This peak is significantly weakened upon reduction. The transformations of these two peaks reveal that the reduction process is efficient. The deconvoluted $\mathrm{N}$ 1s spectra of these composites exhibit two distinct peaks. The dominant peak at $399.0 \mathrm{eV}$ represents the neutral amine nitrogen structure (-NH-), and the tail peak at $401.0 \mathrm{eV}$ can be assigned to positively charged nitrogen ${ }^{43-44}$. The intensity of the high-binding- 
energy (BE) tail peak is weakened upon reduction, indicating that the ascorbic acid has also slightly reduced the doped polypyrrole, which agrees with the analysis of the Raman spectra.

\subsection{Electrochemical testing}

The discharge performances of the batteries with PPy and PPy/RGO cathodes in two types of electrolyte, phosphate buffered saline (PBS) and simulated body fluid (SBF), were tested at different discharge current rates, and the results are shown in Figure 6 (the discharge current densities for battery with PPy/RGO electrodes were calculated based on the total mass of the PPy and RGO components). Each discharge step at a certain discharge current density was set at 300 minutes. It is obvious that the batteries with PPy/RGO cathodes reveal significantly better discharge performance in both of the electrolytes. Generally, they display higher and more stable discharge plateaus and less potential decline. On the contrary, the batteries containing the PPy fibre cathodes suffer from almost linear potential drops.

Fig. 7 presents the dependence of the energy density upon the discharge current rate, which also confirms the superiority of the PPy/RGO cathode. The energy density is calculated based on the equation below,

$$
\mathrm{E}=1 / 2 \text { It } \mathrm{V}^{2}
$$

Where $I$ is the discharge current density, $t$ is the discharge time and $\mathrm{V}$ is the plateau voltage. The batteries with PPy/RGO cathode display the highest energy density of up to $264 \mathrm{mWh} \mathrm{g}^{-1}$ in PBS buffer solution and $210 \mathrm{mWh} \mathrm{g}^{-1}$ in SBF, while it is only $139 \mathrm{mWh} \mathrm{g}^{-1}$ and $129 \mathrm{mWh} \mathrm{g}^{-1}$ for batteries with PPy fibre cathodes, respectively. These results also suggest that the batteries with 1 
g PPy/RGO cathode in SBF electrolyte are capable of powering a neural stimulator continuously for at least 9 hours ${ }^{8}$.

The effects of different electrolytes on battery discharge performance are relatively less distinctive. It is notable, however, that the open circuit potentials and discharge plateaus of batteries with the two cathode materials are slightly higher in PBS. The discharge potential drops at higher current densities in the batteries with PBS electrolyte are much more severe, however, than for the ones with SBF electrolyte. This can be mainly ascribed to the less stable interface reaction on the zinc anode in PBS solution ${ }^{28}$.

The role of the cathode material can generally be explained as that of the oxygen reduction catalyst. Molecular oxygen can be absorbed by the carbon atoms on the polymer chain to form an "oxygen-conductive polymer" bridging complex, which is subsequently reduced to release oxygen intermediates, which eventually form hydroxyl groups ${ }^{32,45}$. This process is associated with the redox reaction of polypyrrole. The redox reaction of polypyrrole is highly reversible, and the polypyrrole can catalyse the ORR continuously until it loses its redox activity ${ }^{24,28,46}$ [Equations (1) and (2)].

$$
\begin{aligned}
& \mathrm{PPy}^{+}+\mathrm{e}^{-} \leftrightarrow \mathrm{PPy}^{0} \\
& \mathrm{O}_{2}+2 \mathrm{e}^{-}+2 \mathrm{H}_{2} \mathrm{O} \rightarrow 2 \mathrm{OH}^{-}
\end{aligned}
$$

Therefore, the reversibility and capability of polypyrrole in the redox process is critical to maintain the ORR catalytic activity of the cathode. To investigate the redox activities of the cathode materials, cyclic voltammetry was conducted on both the pure PPy fibre and the PPy/RGO composite in PBS solution and SBF, respectively, as shown in Figure 8(a) and (b). It is obvious that the redox capacitance of the PPy/RGO composite is significantly higher than that 
of the pure PPy fibre, as indicated by its larger current response. This phenomenon can be ascribed to the synergic effect between the graphene plane and the polypyrrole fibre. The graphene plane provides fast electron transportation channels, which accelerate the ion exchange process in the polypyrrole by providing more free electrons ${ }^{47}$. In PBS electrolyte, the PPy fibre displays two broad oxidation peaks at $-0.43 \mathrm{~V}$ and $-0.08 \mathrm{~V}$, and two reduction peaks at $-0.58 \mathrm{~V}$ and $-0.14 \mathrm{~V}$. These peaks are all shifted slightly to the negative potential for PPy/RGO composite, as labelled in Figure 8(a). This redox pair can be assigned to the cation expulsion and insertion processes of the polypyrrole matrix, which can be demonstrated by the equations below:

$$
\begin{aligned}
& (\mathrm{PPy})_{\mathrm{n}}^{0} \mathrm{~A}_{\mathrm{L}}^{-} \mathrm{C}_{\mathrm{s}}^{+}-e \rightarrow(\mathrm{PPy})_{\mathrm{n}}^{+} \mathrm{A}_{\mathrm{L}}^{-}+\mathrm{C}_{\mathrm{s}}^{+} \\
& (\mathrm{PPy})_{\mathrm{n}}^{+} \mathrm{A}_{\mathrm{L}}^{-} \mathrm{C}_{\mathrm{s}}^{+}+e \rightarrow(\mathrm{PPy})_{\mathrm{n}}^{0} \mathrm{~A}_{\mathrm{L}}^{-}+\mathrm{C}_{\mathrm{s}}^{+}
\end{aligned}
$$

where $A_{L}{ }^{-}$represents large-size anions (e.g., sulfonic acid groups in the buffer) and $C_{s}^{-}$means small size cations $\left(\mathrm{Na}^{+}, \mathrm{K}^{+}\right.$, etc. $)$. It is notable that the voltage difference between the the second reduction/oxidation peak pair becomes narrower for PPy/RGO composite, suggesting its better redox reversibility ${ }^{48}$. Therefore, the batteries with PPy/RGO cathodes displayed a higher and more stable discharge plateau, which is mainly attributed to its higher ORR catalytic efficiency, which originates from better redox reversibility and higher redox capacitance.

The nature of the electrolyte can affect the redox capability of the cathode material and consequently determine its catalytic efficiency as well. In PBS solution, the shapes of CV curves are nearly rectangular, representing the large capacitance. The CV curves in SBF, however, generally display spindle-like shapes with a significant reduction in capacitance. This is mainly due to the presence of large molecules such as HEPEs (4-(2-hydroxyethyl)-1- 
piperazineethanesulfonic acid) which can block the charge transfer channels along the PPy matrix $^{49}$. Due to this effect, the open circuit potentials of batteries with SBF electrolyte are significantly lower, and the charge potential drops are much more severe.

To further investigate the interfacial electrochemical behaviour of the cathodes in these two types of electrolyte, electrochemical impedance spectroscopy (EIS) was conducted after 3 hours of discharge. The Nyquist plots reflecting the relationship of the imaginary impedance -Z" versus the real impedance $Z^{\prime}$ are shown in Figure 9(a) and (b). The Nyquist plots mainly include a semicircular region lying on the real axis, which is followed by a straight line. The semicircle observed at high frequencies usually corresponds to the Faradaic charge carrier transfer process, and its radius can reflect the charge transfer resistance of the redox processes, whereas the linear part in the low frequency range represents the diffusion-controlled electrode process ${ }^{50}$. The EIS spectra were simulated by the equivalent circuit as showed in the insets of Figure.4. Such equivalent circuits composed of four major components, including the bulk solution resistance R1, the internal resistance R2, the electron double layer capacitance C1 and the Warburg diffusion element W1. The values of each elements were calculated and listed in Table.2. It is obvious that the PPy/RGO electrodes present lower charge transfer resistance in these two electrolytes, which provides an explanation for its superior discharge performance. Meanwhile, the nature of the electrolyte can affect the electrochemical processes by affecting the diffusion process and Faradaic charge carrier transfer process spontaneously. According to the results shown in Figure 9, the Warburg diffusion elements are generally larger in PBS solution for both types of electrode, indicating the ion diffusion process is faster in the PBS solution. The bulk solution resistance is about $9.5 \Omega$ for PBS solution and $24.5 \Omega$ for SBF, respectively ${ }^{51}$. The lower 
solution resistance in PBS can also be ascribed to better ion mobility in the electrolyte. Low ion mobility can decrease the ion exchange rate at the interfaces of both the anode and the cathode, which consequently degrades the catalytic efficiency of the cathode and simultaneously, the ion release rate at the zinc anode. Thus, it can be inferred that the open circuit potential and discharge plateaus of a battery with SBF electrolyte is generally lower than the one with SBF electrolyte. This can also explain why the batteries with SBF electrolyte have lower open circuit potential and lower capacities.

\section{CONCLUSION}

Polypyrrole fibre/RGO composite was successfully synthesized via a simple one-step chemical polymerization method. A microstructure of fine and uniform polypyrrole fibres decorated on 2D RGO sheets was obtained. The 2D graphene planes provide faster electron transfer channels and protect the polypyrrole fibres from rapid decay in redox capability. Hence, the PPy/RGO composite as cathode displayed better redox capability and reversibility than the pure PPy fibre cathode. This composite also has high electric conductivity and large specific surface area. Due to these structural and electrochemical property advantages, the composite exhibited higher electrocatalytic activity towards the oxygen reduction reaction than the pure polypyrrole nanofibres. Thus, the batteries constructed with this composite material have stable energy outputs at both low and high current densities. The nature of the electrolyte also affects the battery performance with SBF or PBS as electrolyte. SBF contains large organic molecules, which can cause the solution resistance to increase, which will consequently retard the interface reactions at both the anode and cathode. The battery with PPy/RGO cathode achieved an energy 
density of $264 \mathrm{mWh} \mathrm{g}^{-1}$ in PBS and $210 \mathrm{mWh} \mathrm{g}^{-1}$ in SBF, which suggests that it is feasible for powering a broad range of implantable medical microdevices.

\section{ACKNOWLEDGMENTS}

The authors acknowledge the Australian Research Council (ARC) for financial support under the ARC Centre of Excellence for Electromaterials Science and the Electron Microscopy Centre at the University of Wollongong for providing the electron microscopy facilities.

\section{REFERENCES}

1. Mallela, V. S.; Ilankumaran, V.; Rao, N. S., Trends in Cardiac Pacemaker Batteries. Indian pacing and electrophysiology journal 2004, 4 (4), 201.

2. $\quad$ Takeuchi, E. S.; Leising, R. A., Lithium Batteries for Biomedical Applications. MRS bulletin 2002, 27 (08), 624-627.

3. Greatbatch, W.; Lee, J. H.; Mathias, W.; Eldridge, M.; Moser, J. R.; Schneider, A. A., The Solid-state Lithium Battery: A New Improved Chemical Power Source for Implantable Cardiac Pacemakers. Biomedical Engineering, IEEE Transactions on 1971, (5), 317-324.

4. $\quad$ Crespi, A. M.; Somdahl, S. K.; Schmidt, C. L.; Skarstad, P. M., Evolution of Power Sources for Implantable Cardioverter Defibrillators. Journal of power sources 2001, 96 (1), 3338.

5. Goto, K.; Nakagawa, T.; Nakamura, O.; Kawata, S., An Implantable Power Supply with an Optically Rechargeable Lithium Battery. Biomedical Engineering, IEEE Transactions on 2001, 48 (7), 830-833.

6. Jimbo, H.; Miki, N., Gastric-fluid-utilizing Micro Battery for Micro Medical Devices. Sensors and Actuators B: Chemical 2008, 134 (1), 219-224.

7. Schulman, J. H.; Maltan, A. A.; Santogrossi, T. A., Zinc Air Battery and its Uses. Google Patents: 2005.

8. Wei, X.; Liu, J., Power Sources and Electrical Recharging Strategies for Implantable medical devices. Frontiers of Energy and Power Engineering in China 2008, 2 (1), 1-13.

9. Holmes, C. F., The Role of Lithium Batteries in Modern Health Care. Journal of power sources 2001, 97, 739-741.

10. Schmidt, C. L.; Skarstad, P. M., The Future of Lithium and Lithium-ion Batteries in Implantable Medical Devices. Journal of power sources 2001, 97, 742-746. 
11. Powers, R. A., Batteries for Low Power Electronics. Proceedings of the IEEE 1995, 83 (4), 687-693.

12. Dell, R.; Gord, J.; Schulman, J., Battery-powered Patient Implantable Device. Google Patents: 2003.

13. Lavan, D. A.; McGuire, T.; Langer, R., Small-scale Systems for In-vivo Drug Delivery. Nature biotechnology 2003, 21 (10), 1184-1191.

14. Darwish, A.; Hassanien, A. E., Wearable and Implantable Wireless Sensor Network Solutions for Healthcare Monitoring. Sensors 2011, 11 (6), 5561-5595.

15. Meng, C.; Gall, O. Z.; Irazoqui, P. P., A Flexible Super-capacitive Solid-state Power Supply for Miniature Implantable Medical Devices. Biomedical microdevices 2013, 15 (6), 973983.

16. Rasouli, M.; Phee, L. S. J., Energy Sources and their Development for Application in Medical Devices. Expert review of medical devices 2010, 7 (5), 693-709.

17. Heller, A., Potentially Implantable Miniature Batteries. Analytical and bioanalytical chemistry 2006, 385 (3), 469-473.

18. Fosmire, G. J., Zinc Toxicity. The American journal of clinical nutrition 1990, 51 (2), 225-227.

19. Gu, X.; Zheng, Y.; Cheng, Y.; Zhong, S.; Xi, T., In Vitro Corrosion and Biocompatibility of Binary Magnesium Alloys. Biomaterials 2009, 30 (4), 484-498.

20. Lee, K. B., Urine-activated Paper Batteries for Biosystems. Journal of Micromechanics and Microengineering 2005, 15 (9), S210.

21. Lee, K. B., Two-step Activation of Paper Batteries for High Power Generation: design and fabrication of biofluid-and water-activated paper batteries. Journal of Micromechanics and Microengineering 2006, 16 (11), 2312.

22. Pushparaj, V. L.; Shaijumon, M. M.; Kumar, A.; Murugesan, S.; Ci, L.; Vajtai, R.; Linhardt, R. J.; Nalamasu, O.; Ajayan, P. M., Flexible Energy Storage Devices Based on Nanocomposite Paper. Proceedings of the National Academy of Sciences 2007, 104 (34), 1357413577.

23. Lee, J. S.; Tai Kim, S.; Cao, R.; Choi, N. S.; Liu, M.; Lee, K. T.; Cho, J., Metal-air Batteries with High Energy Density: Li-air versus Zn-air. Advanced Energy Materials 2011, 1 (1), 34-50.

24. Winther-Jensen, B.; Gaadingwe, M.; Macfarlane, D.; Forsyth, M., Control of Magnesium Interfacial Reactions in Aqueous Electrolytes towards a Biocompatible Battery. Electrochimica Acta 2008, 53 (20), 5881-5884.

25. Kong, Y.; Wang, C.; Yang, Y.; Too, C. O.; Wallace, G. G., A Battery Composed of a Polypyrrole Cathode and a Magnesium Alloy Anode-Toward a bioelectric battery. Synthetic Metals 2012, 162 (7), 584-589.

26. Zhang, T.; Tao, Z.; Chen, J., Magnesium-air Batteries: From Principle to Application. Materials Horizons 2014.

27. Li, S.; Liu, H.-K.; Wang, C.; Guo, Z.; Wallace, G., Flexible Cellulose based PolypyrroleMultiwalled Carbon Nanotube Films for Bio-compatible Zinc Battery Activated by Simulated Body Fluid. J. Mater. Chem. A 2013.

28. Li, S.; Sultana, I.; Guo, Z.; Wang, C.; Wallace, G.; Liu, H.-K., Polypyrrole as Cathode Materials for Zn-polymer Battery with Various Biocompatible Aqueous Electrolytes. Electrochimica Acta 2013, 95, 212-217. 
29. Qu, L.; Liu, Y.; Baek, J.-B.; Dai, L., Nitrogen-doped Graphene as Efficient Metal-free Electrocatalyst for Oxygen Reduction in Fuel Cells. Acs Nano 2010, 4 (3), 1321-1326.

30. Yang, Z.; Yao, Z.; Li, G.; Fang, G.; Nie, H.; Liu, Z.; Zhou, X.; Chen, X. a.; Huang, S., Sulfur-doped Graphene as an Efficient Metal-free Cathode Catalyst for Oxygen Reduction. Acs Nano 2011, 6 (1), 205-211.

31. Geng, D.; Chen, Y.; Chen, Y.; Li, Y.; Li, R.; Sun, X.; Ye, S.; Knights, S., High OxygenReduction Activity and Durability of Nitrogen-doped Graphene. Energy Environ. Sci. 2011, 4 (3), 760-764.

32. Jiao, Y.; Zheng, Y.; Jaroniec, M.; Qiao, S. Z., Origin of the Electrocatalytic Oxygen Reduction Activity of Graphene-based Catalysts: A Roadmap to Achieve the Best Performance. Journal of the American Chemical Society 2014, 136 (11), 4394-4403.

33. Z Zhang, X.; Zhang, J.; Song, W.; Liu, Z., Controllable Synthesis of Conducting Polypyrrole Nanostructures. The Journal of Physical Chemistry B 2006, 110 (3), 1158-1165. 34. Xu, Y.; Bai, H.; Lu, G.; Li, C.; Shi, G., Flexible Graphene Films via the Filtration of Water-soluble Noncovalent Functionalized Graphene Sheets. Journal of the American Chemical Society 2008, 130 (18), 5856-5857.

35. Oyane, A.; Kim, H. M.; Furuya, T.; Kokubo, T.; Miyazaki, T.; Nakamura, T., Preparation and Assessment of Revised Simulated Body Fluids. Journal of Biomedical Materials Research Part A 2003, 65 (2), 188-195.

36. Liu, Y.-C.; Hwang, B.-J.; Jian, W.-J.; Santhanam, R., In situ Cyclic Voltammetrysurface-enhanced Raman Spectroscopy: studies on the doping-undoping of polypyrrole film. Thin Solid Films 2000, 374 (1), 85-91.

37. Santos, M.; Brolo, A.; Girotto, E., Study of Polaron and Bipolaron States in Polypyrrole by In-situ Raman Spectroelectrochemistry. Electrochimica acta 2007, 52 (20), 6141-6145.

38. Xu, C.; Sun, J.; Gao, L., Synthesis of Novel Hierarchical Graphene/polypyrrole Nanosheet Composites and Their Superior Electrochemical Performance. Journal of Materials Chemistry 2011, 21 (30), 11253-11258.

39. Dresselhaus, M. S.; Jorio, A.; Hofmann, M.; Dresselhaus, G.; Saito, R., Perspectives on Carbon Nanotubes and Graphene Raman Spectroscopy. Nano letters 2010, 10 (3), 751-758.

40. Zhou, Y.; Bao, Q.; Tang, L. A. L.; Zhong, Y.; Loh, K. P., Hydrothermal Dehydration for the "green" Reduction of Exfoliated Graphene Oxide to Graphene and Demonstration of Tunable Optical Limiting Properties. Chemistry of Materials 2009, 21 (13), 2950-2956.

41. Zhu, Y.; Stoller, M. D.; Cai, W.; Velamakanni, A.; Piner, R. D.; Chen, D.; Ruoff, R. S., Exfoliation of Graphite Oxide in Propylene Carbonate and Thermal Reduction of the Resulting Graphene Oxide Platelets. Acs Nano 2010, 4 (2), 1227-1233.

42. Gao, J.; Liu, F.; Liu, Y.; Ma, N.; Wang, Z.; Zhang, X., Environment-friendly Method to Produce Graphene that Employs Vitamin C and Amino acid. Chemistry of Materials 2010, 22 (7), 2213-2218.

43. Malitesta, C.; Losito, I.; Sabbatini, L.; Zambonin, P. G., New findings on Polypyrrole Chemical Structure by XPS Coupled to Chemical Derivatization Labelling. Journal of Electron Spectroscopy and Related Phenomena 1995, 76, 629-634.

44. Kang, E.; Neoh, K.; Tan, K., The Intrinsic Redox States in Polypyrrole and Polyaniline: A Comparative Study by XPS. Surface and interface analysis 1992, 19 (1 - 12), 33-37. 45. Khomenko, V.; Barsukov, V.; Katashinskii, A., The Catalytic Activity of Conducting Polymers toward Oxygen Reduction. Electrochimica Acta 2005, 50 (7), 1675-1683. 
46. Wu, A.; Venancio, E. C.; MacDiarmid, A. G., Polyaniline and Polypyrrole Oxygen Reversible Electrodes. Synthetic Metals 2007, 157 (6), 303-310.

47. Hou, J.; Shao, Y.; Ellis, M. W.; Moore, R. B.; Yi, B., Graphene-based Electrochemical Energy Conversion and Storage: fuel cells, supercapacitors and lithium ion batteries. Physical Chemistry Chemical Physics 2011, 13 (34), 15384-15402.

48. Jin, C.; Yang, F.; Yang, W., Electropolymerization and Ion Exchange Properties of a Polypyrrole film Doped by Para - toluene Sulfonate. Journal of applied polymer science 2006, 101 (4), 2518-2522.

49. Miller, D. L.; Bockris, J. O. M., Structure of the Polypyrrole/Solution Interphase. Journal of the Electrochemical Society 1992, 139 (4), 967-976.

50. Macdonald, J. R.; Barsoukov, E., Impedance Spectroscopy: Theory, Experiment, and Applications. History 2005, 1, 8.

51. Liu, C.; Bi, Q.; Leyland, A.; Matthews, A., An Electrochemical Impedance Spectroscopy study of the Corrosion Behaviour of PVD Coated Steels in $0.5 \mathrm{~N} \mathrm{NaCl}$ Aqueous Solution: Part II.: EIS Interpretation of Corrosion Behaviour. Corrosion Science 2003, 45 (6), 1257-1273. 
Figure.1

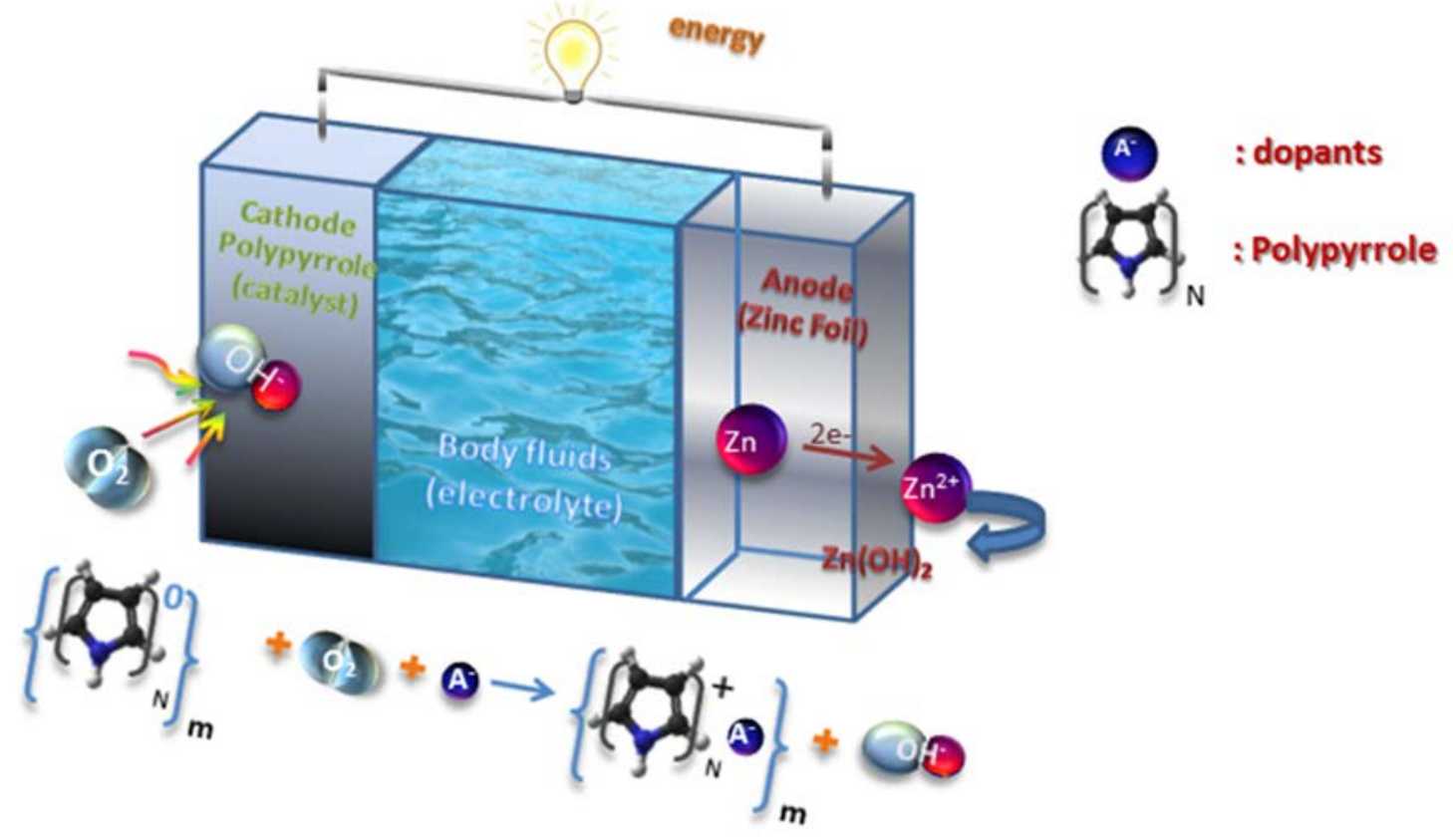

Figure.2

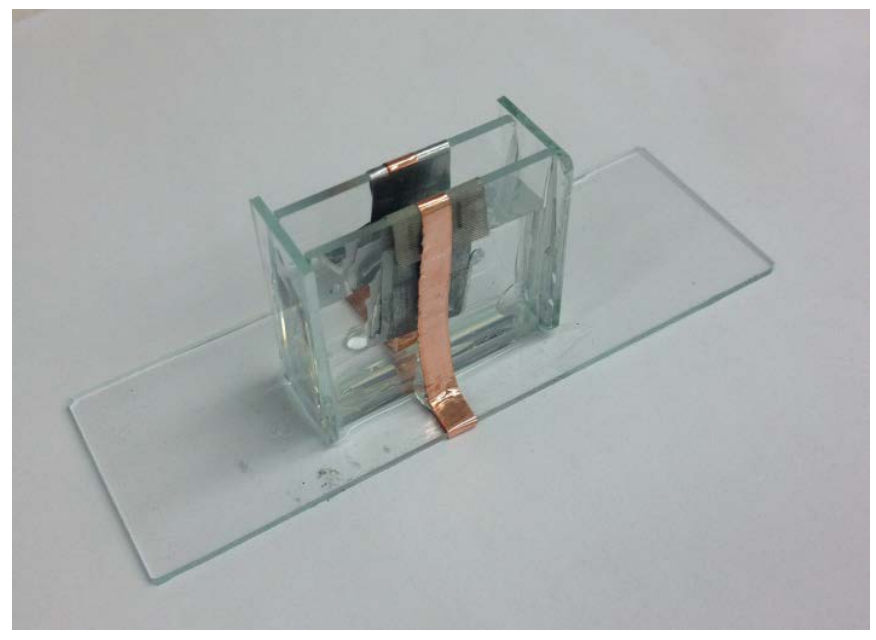


Figure.3

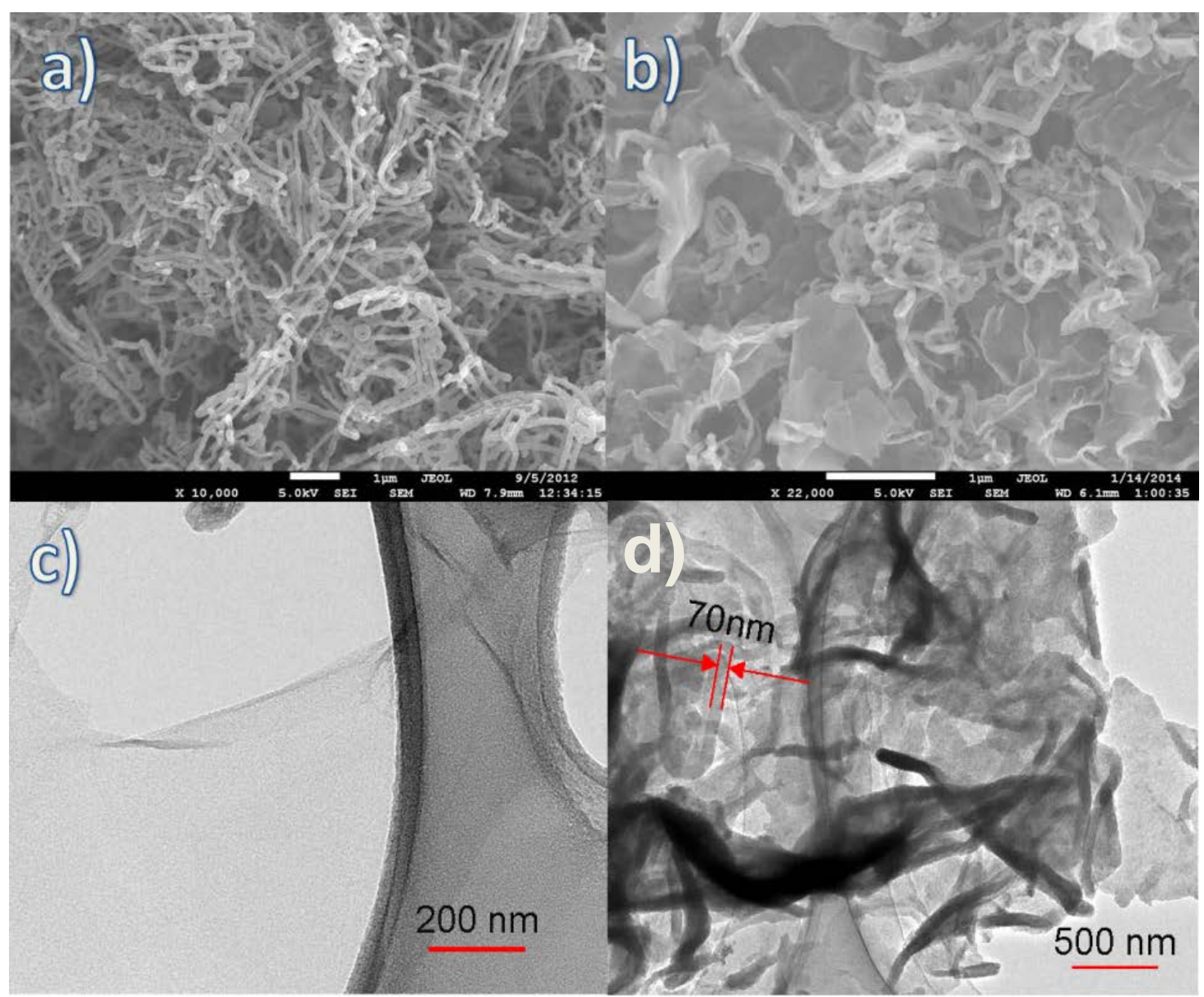


Figure .4
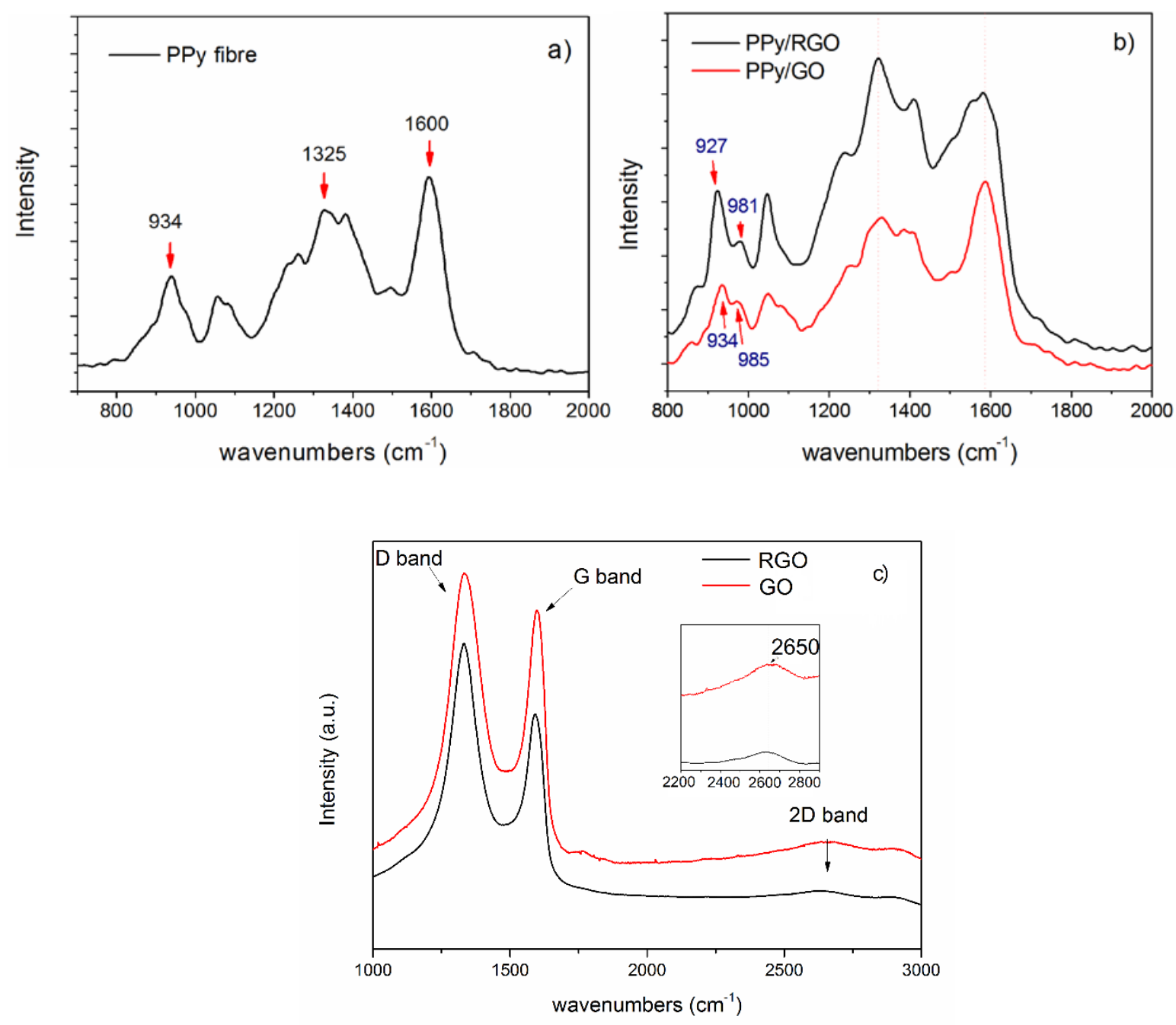
Figure 5.
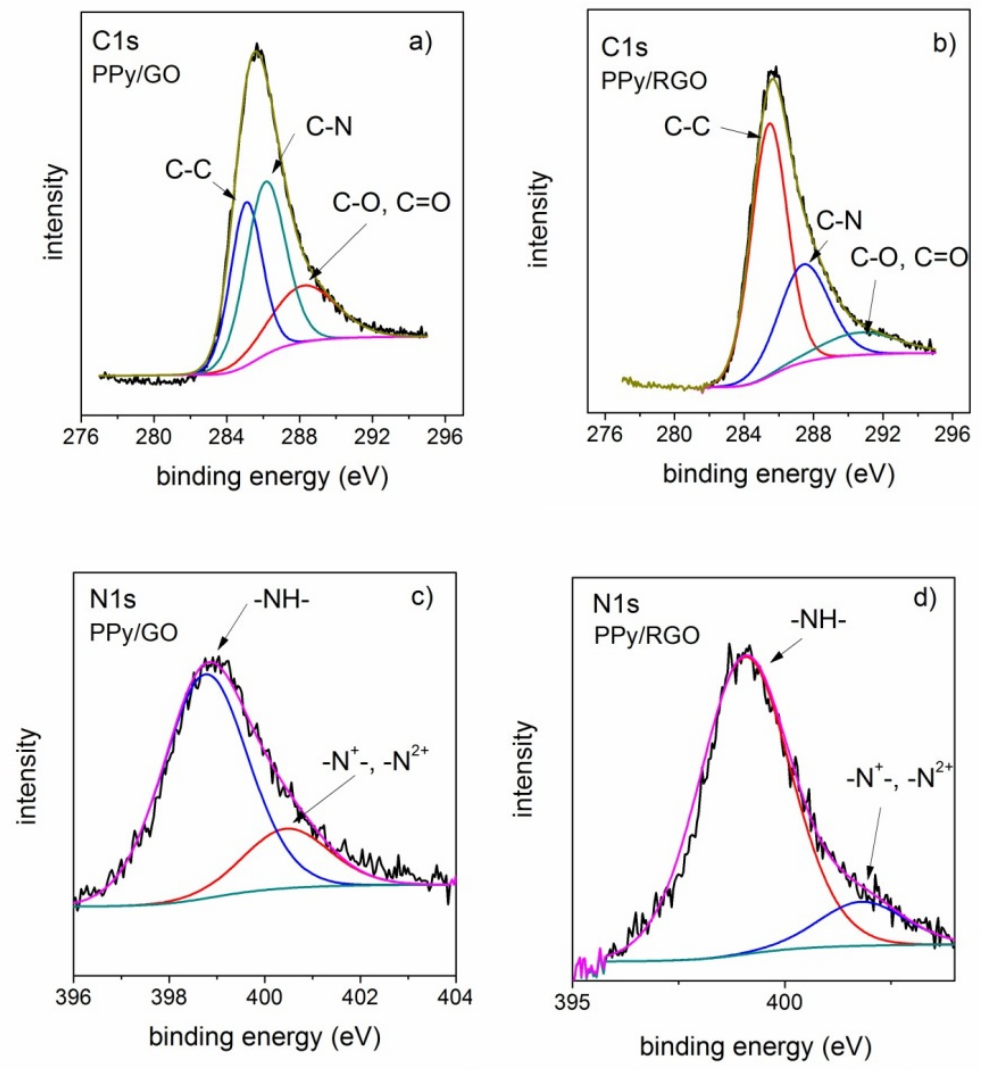

Figure 6.
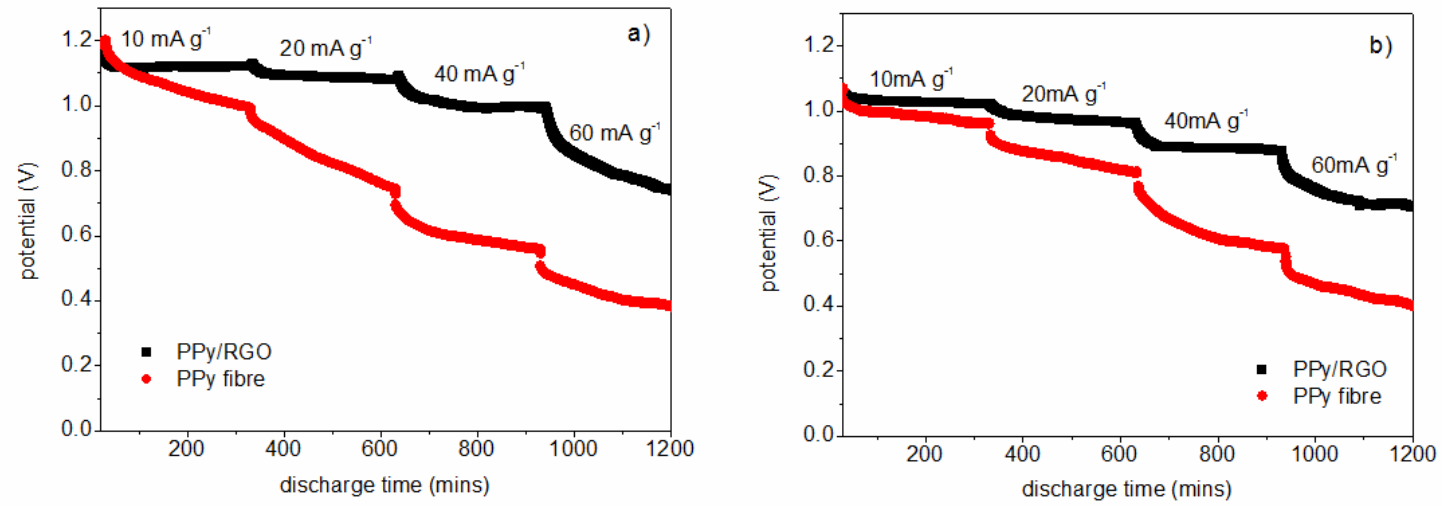
Figure 7.
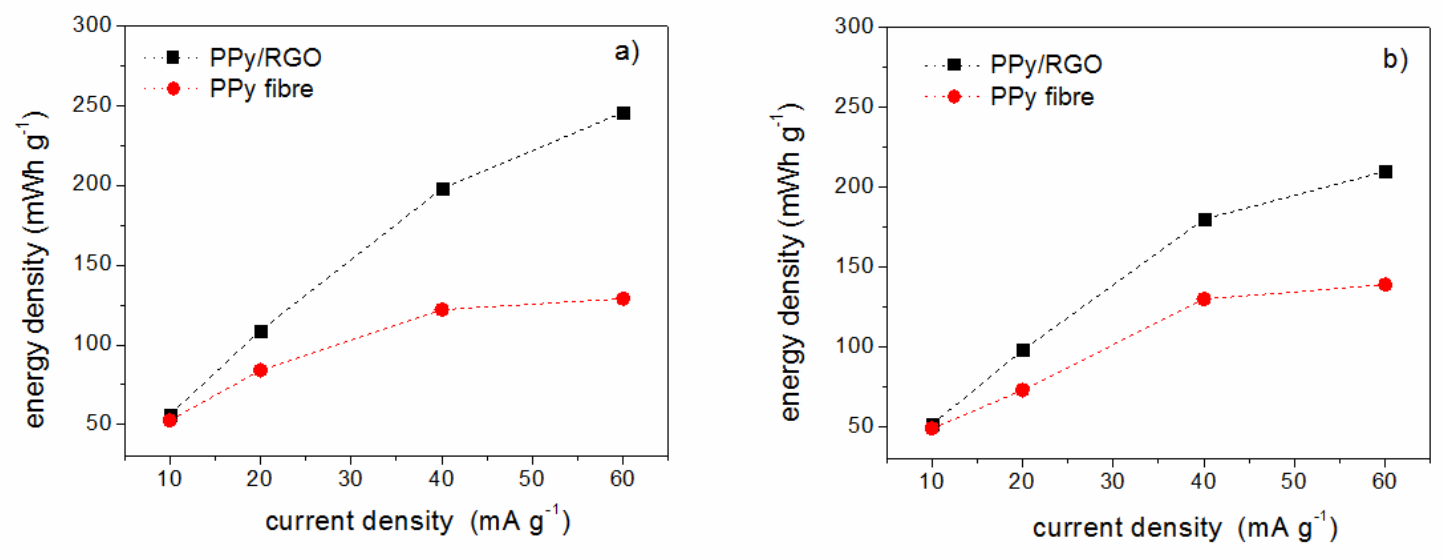

Figure 8
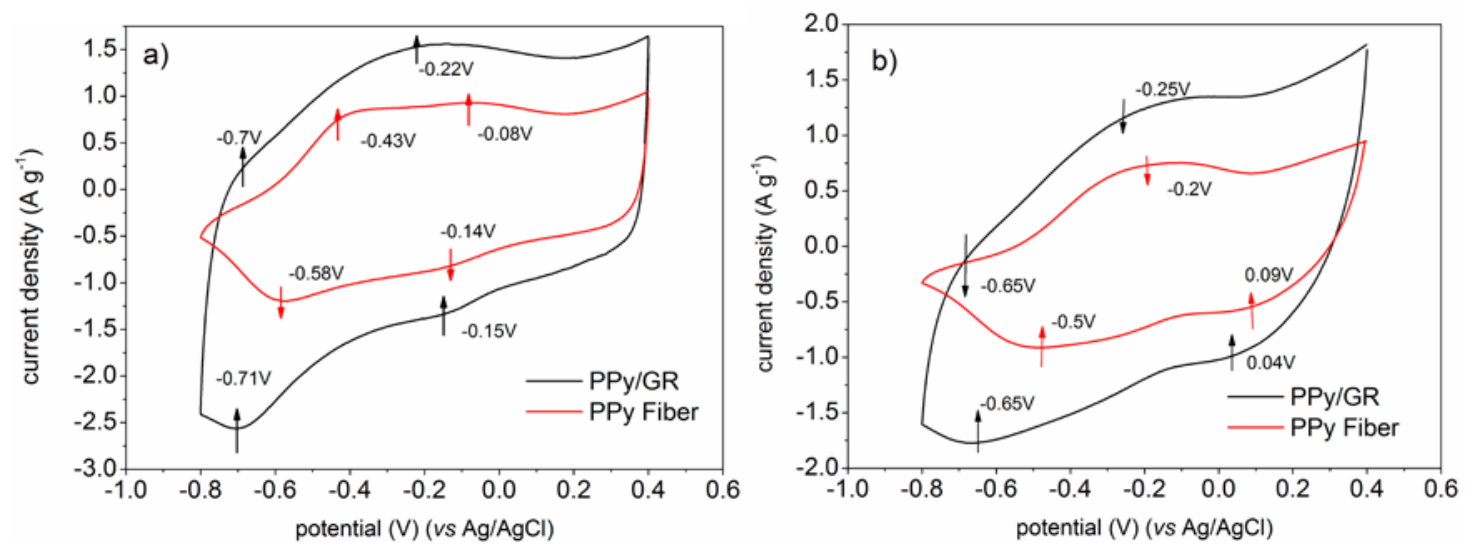
Figure 9
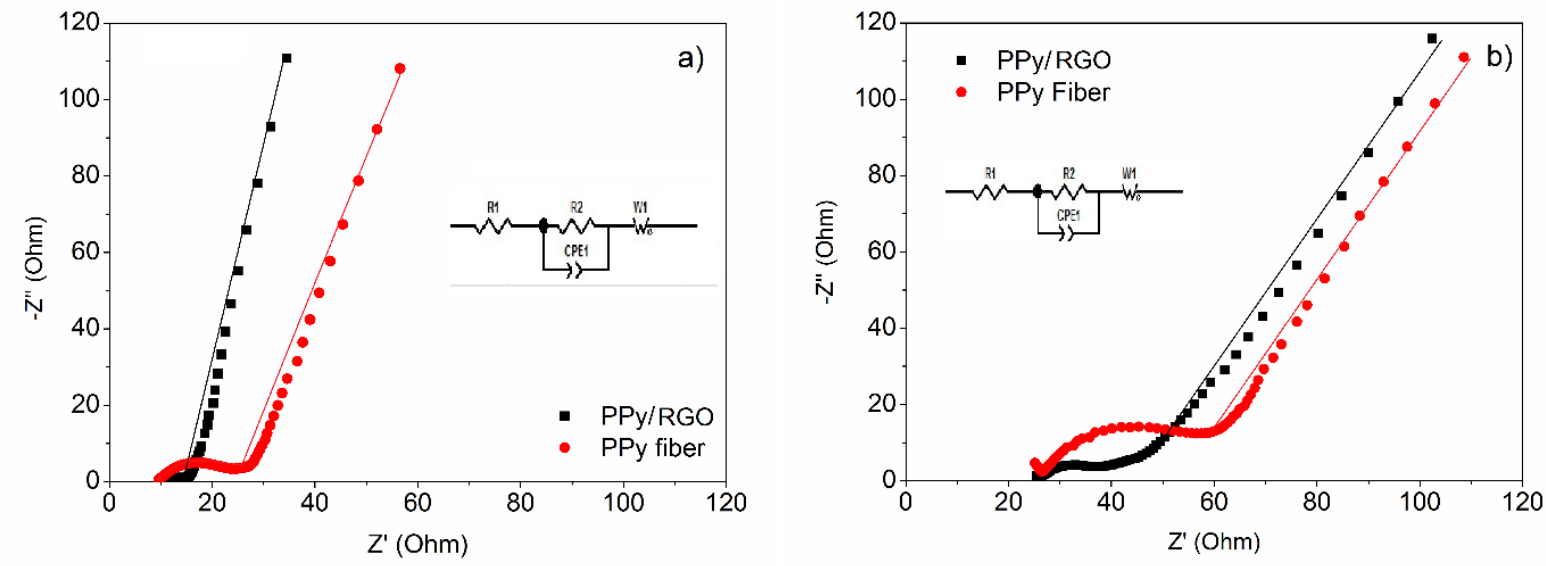

Table 1.

\begin{tabular}{|l|l|l|l|l|l|l|l|l|l|}
\hline Ions(mM) & $\mathbf{N a}^{+}$ & $\mathbf{K}^{+}$ & $\mathbf{M g}^{2+}$ & $\mathbf{C a}^{2+}$ & $\mathbf{C l}^{-}$ & $\mathbf{H C O}^{3-}$ & $\mathbf{H P O}_{4}{ }^{2-}$ & $\mathbf{S O}_{4}{ }^{2-}$ & buffer \\
\hline r-SBF & 142.0 & 5.0 & 1.5 & 2.5 & 103.0 & 27.0 & 1.0 & 0.5 & 11.928 \\
\hline Blood plasma & 142.0 & 5.0 & 1.5 & 2.5 & 103.0 & 27.0 & 1.0 & 0.5 & - \\
\hline
\end{tabular}

Table 2.

\begin{tabular}{|l|l|l|l|l|}
\hline & R1 & R2 & Cp1 & W1 \\
\hline Ppy fibre/PBS & 9.63 & 8.64 & 0.76 & 15.4 \\
\hline Ppy RGO/PBS & 9.48 & 3.95 & 0.69 & 4.86 \\
\hline Ppy fibre/SBF & 24.52 & 20.6 & 0.77 & 40.1 \\
\hline Ppy RGO/SBF & 24.41 & 8.59 & 0.68 & 42.3 \\
\hline
\end{tabular}


Figure captions

\section{FIGURES CAPTIONS}

Figure.1 working principle scheme of an implantable Zinc-air battery

Figure.2 a digital photograph of a fully constructed battery

Figure.3 Field emission electron scanning microscope (FESEM) image of (a) pure polypyrrole fibre and (b) polypyrrole fibre/RGO composite; Transmission electron microscope (TEM) images of (c) pure RGO and (d) polypyrrole fibre/RGO composite

Figure.4 Raman spectrums of (a) PPy fibre (b) PPy fibre/RGO composite and PPy fibre/GO composite (c) RGO and GO (for comparison)

Figure.5 De-convoluted C1s and N1s X-ray photoelectron spectroscopy (XPS) spectra of PPy fiber/GO composite (a, c) and PPy fiber/RGO composite (b, d)

Figure.6 the discharge curves of batteries composed of both Pure PPy fiber and PPy/RGO electrodes being tested in (a) the 0.2M PBS solution and (b) SBF solution.

Figure.7 energy densities versus discharge current density of battery with PPy/RGO and PPy fibre cathode in different electrolyte (a) 0.2M PBS solution and (b) SBF

Figure.8 The cyclic voltammetry curves of PPy/GR and Pure PPy fibre cathodes in (a) 0.2M PBS solution and (b) SBF (scan rate $=5 \mathrm{mV} \mathrm{S}^{-1}$ )

Figure 9. EIS spectra and the simulated spectra (lines) of PPy/RGO and pure PPy fiber tested in (a) $0.2 \mathrm{M}$ PBS solution and (b) SBF solution. (Inset, equivalent circuits)

Table titles

Table.1 The ion concentrations comparison between the revised simulated body fluid (r-SBF) and blood plasma

Table.2 The fitting values of the equivalent circuit elements 
Table of content graphic

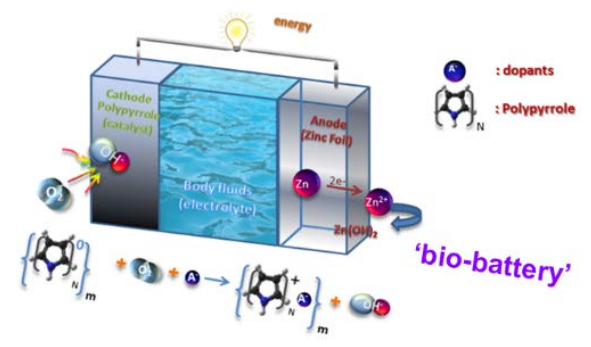

